\title{
Subsurface In Situ Elemental Composition Measurements with PING
}

\author{
Ann Parsons, Timothy McClanahan \\ NASA/ Goddard Space Flight Ctr. \\ 8800 Greenbelt Rd, Code 691 \\ Greenbelt, MD 20771 \\ 301-286-1107 \\ Ann.M.Parsons@nasa.gov \\ Timothy.P.McClanahan@nasa.gov
}

\author{
Suzanne Nowicki \\ University of Michigan \\ NASA/ Goddard Space Flight Ctr. \\ 8800 Greenbelt Rd, Code 691 \\ Greenbelt, MD 20771 \\ 301-614-7034 \\ Suzanne.F.Nowicki@nasa.gov
}

\author{
Julia Bodnarik \\ Vanderbilt University \\ NASA/ Goddard Space Flight Ctr. \\ 8800 Greenbelt Rd, Code 691 \\ Greenbelt, MD 20771 \\ 301-286-1398 \\ Julia.G.Bodnarik@nasa.gov
}

\author{
Jeffrey Schweitzer \\ University of Connecticut \\ 2152 Hillside Road, U-3046 \\ Storrs, CT 06269-3046 \\ 860-486-6010 \\ schweitz@phys.uconn.edu
}

\author{
Larry Evans \\ Computer Sciences Corporation \\ NASA/ Goddard Space Flight Ctr. \\ 8800 Greenbelt Rd, Code 691 \\ Greenbelt, MD 20771 \\ 301-286-5759 \\ Larry.Evans@nasa.gov
}

\author{
Richard Starr \\ Catholic University of America \\ NASA/ Goddard Space Flight Ctr. \\ 8800 Greenbelt Rd, Code 691 \\ Greenbelt, MD 20771 \\ 301-286-5073 \\ Richard.Starr@nasa.gov
}

\begin{abstract}
This paper describes the Probing In situ with Neutron and Gamma rays (PING) instrument, that can measure the subsurface elemental composition in situ for any rocky body in the solar system without the need for digging into the surface. PING consists of a Pulsed Neutron Generator (PNG), a gamma ray spectrometer and neutron detectors. Subsurface elements are stimulated by high-energy neutrons to emit gamma rays at characteristic energies. This paper will show how the detection of these gamma rays results in a measurement of elemental composition. Examples of the basalt to granite ratios for aluminum and silicon abundance are provided.
\end{abstract}

\section{TABLE OF CONTENTS}

1. INTRODUCTION 1

\section{ACtive Gamma Ray/ NeUtron}

INSTRUMENTS .2

3. PING EXPERIMENTS .....................................3

4. Preliminary Results ................................5

5. FUTURE WORK .................................................6

6. CONCLUSIONS ..................................................... 7

REFERENCES................................................7

\section{INTRODUCTION}

The Probing In situ with Neutrons and Gamma rays (PING) instrument [1] is a promising planetary science application of the active neutron-gamma ray technology that has been used successfully in oil field well logging and mineral exploration on Earth for decades. These techniques can be very powerful for non-invasive in situ measurements of the subsurface elemental composition on other planets. The objective of our active neutron-gamma ray technology program at NASA Goddard Space Flight Center (NASA/GSFC) is to bring instruments using this technology to the point where they can be flown on a variety of surface lander or rover missions to the Moon, Mars, Venus, asteroids, comets and the satellites of the outer planets. PING differs from orbital gamma ray and neutron instruments in that it carries its own source of high energy neutrons: a high intensity Pulsed Neutron Generator (PNG) that produces fast $(14 \mathrm{MeV})$ neutrons at a rate $\sim 100 \mathrm{x}$ greater than neutrons generated via the interaction of galactic cosmic rays with planetary materials. This orders of magnitude higher neutron rate results in significantly shorter data acquisition times for the same precision.

A critical measurement in the investigation of planetary bodies is the determination of the chemical composition of planetary crusts. In the course of planetary evolution, several processes modify the distribution of elements on the surface of a planet, namely mantle and crust differentiation, magma transport and its eruption onto a surface, and impact cratering. The abundance of certain elements associated with different types of geochemical behavior can provide important insight into the origin and evolution of these bodies. The global planetary composition is characteristic of the nature of its formation during the accretion from the solar nebula. In the course of planetary evolution, the elemental distribution is greatly modified by such processes as core and crust formation during differentiation and later magma formation and emplacement. A detailed discussion of the use of geochemical information in the study of the formation and evolution of planetary bodies can be found, for example, in [2,3].

PING will be an important tool for the above scientific investigations since it measures the bulk elemental composition as a function of depth without the need for drilling or digging into the surface. The ability to get this kind of information without drilling has many advantages: physically drilling into a planetary surface adds mass, power, volume and complexity to a mission and can pose great difficulties in fitting into constrained mission budgets. 
Scientifically, the physical process of drilling into the surface causes problems with mixing the materials in various layers and can make depth measurements of elemental composition very difficult. PING can make subsurface measurements without disturbing the surface because of the penetrating nature of both the neutrons and gamma rays.

PING is an adaptation of well-established techniques that have been used in the oil industry for decades. PING is capable of measuring in situ bulk elemental composition and density information over a $\sim 1$ meter radius and down to $50 \mathrm{~cm}$ below a planets surface without the need for digging. While it is quite practical on Earth to take numerous samples of materials and send them to advanced laboratories for complete elemental analysis, it is extremely difficult to obtain samples from the subsurface of other planets in our solar system and bring them to Earth for similar analysis. The detailed analysis of the distribution of elements with depth provides useful information that can often answer questions about the chemical environment, the physical properties of the materials and the evolutionary processes that formed the geological structures. On Earth, the oil industry has led the way in the development of specialized instruments that can be used in the field to provide a depthdependent elemental analysis and measurements of bulk properties that is almost as good as those performed in the laboratory.

\section{Active Gamma Ray/ Neutron INSTRUMENTS}

\subsection{PING Operating Principles}

PING consists of three basic components: 1) a Pulsed Neutron Generator (PNG) that emits intense pulses of fast $(14 \mathrm{MeV})$ neutrons to excite materials at and below the planetary surface, 2) a gamma-ray spectrometer to measure characteristic gamma rays from the excited elements, and 3) neutron detectors to measure the properties of the resulting lower-energy neutrons that reach the surface after scattering. As illustrated in Figure 1, when an extra-terrestrial surface is bombarded with fast neutrons from a neutron generator, the nuclei in the planetary material are excited and emit gamma radiation that is characteristic of isotopes of the elements present in the material. The intensity of these characteristic gamma rays can be directly converted to the abundance of the specific elements that emitted them, assuming an isotopic distribution typical of other bodies in the Solar System such as Earth. Additionally, fast neutrons incident on the planetary surface will lose energy with successive interactions within the material at a rate that depends on the material properties. By measuring the count rates of these slowed neutrons that reach the surface, we obtain information about density, hydrogen content, and subsurface layering. Active instruments

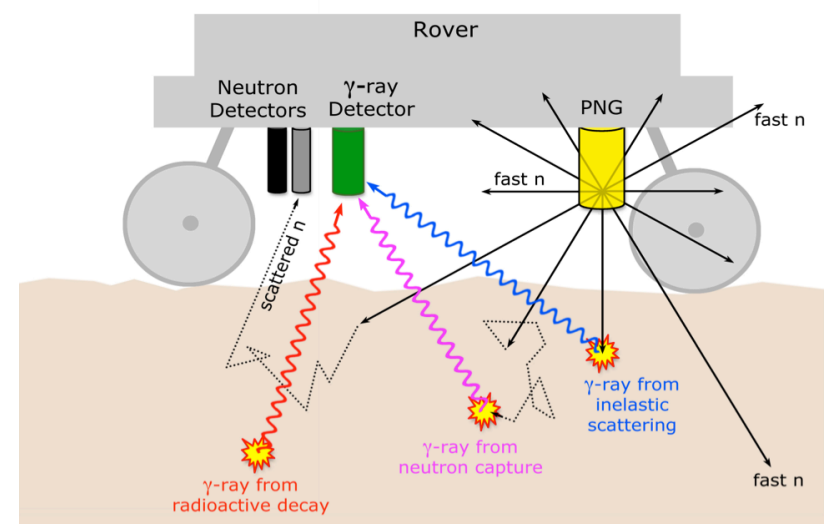

Figure 1: This sketch shows the PING neutron detectors, gamma-ray detector and $P N G$ attached to the bottom of a planetary surface rover and illustrates the physical processes that produce the gamma rays and neutrons that PING detects.

that stimulate planetary surfaces with fast neutrons and then detect both the gamma rays and neutrons emerging from the object's surface thus provide valuable information about the subsurface material composition.

These gamma rays are emitted at specific energies characteristic of each element via processes such as inelastic neutron scattering, thermal neutron capture, delayed activation, and natural radioactivity. Thus the elemental abundances in the stimulated material can be readily determined from analysis of the gamma rays emitted by these different processes. The probability of these processes occurring depends on the speed of the neutrons as they lose energy by scattering from fast $(14 \mathrm{MeV})$ to epithermal (< $500 \mathrm{keV})$ and thermal $(\leq 0.4 \mathrm{eV})$ energies. Since the inelastic neutron scattering process requires the neutron to have a significantly higher energy than the reaction threshold in the nucleus (1-6 MeV), inelastic scattering events occur before the incident $14 \mathrm{MeV}$ neutrons have had time to lose much energy. Thus the emitted gamma rays from inelastic scattering processes are produced earlier in time than gamma rays produced by other processes. Characteristic gamma rays resulting from thermal or epithermal neutron capture interactions appear a short while later. Following the inelastic scattering or neutron capture processes, the interacting nuclei may be left in a radioactive ground state that lives for some time (fraction of a second to

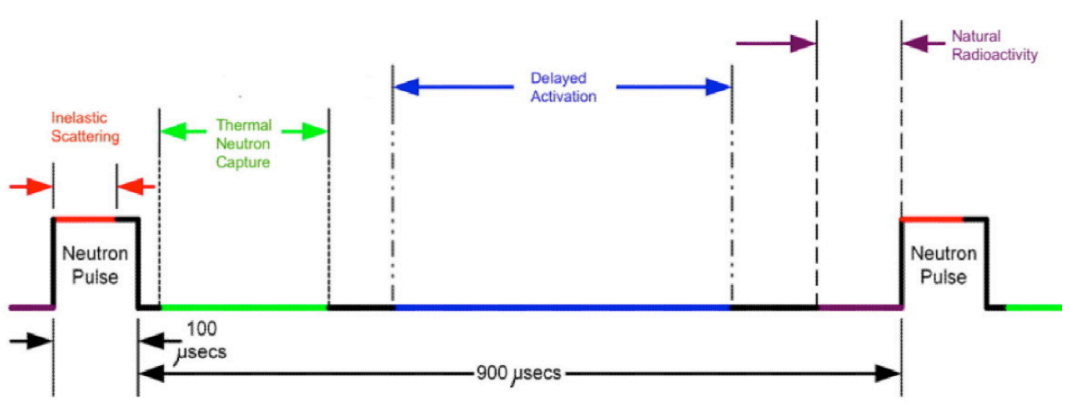

Figure 2: Placement of timing windows relative to each PNG pulse. 
many days) and then usually decays by beta decay, often accompanied by gamma ray emission from the daughter nucleus. Finally, there the naturally radioactive elements K, $\mathrm{Th}$, and $\mathrm{U}$ that are common in solid bodies in the solar system and emit characteristic gamma rays from radioactive decay. No outside stimulation of these elements is needed for gamma-ray production. The intensity of the characteristic gamma-ray lines measured by the gamma-ray spectrometer can thus be used to infer absolute elemental abundances of the material. Elements accessible to PING include $\mathrm{C}, \mathrm{H}, \mathrm{O}, \mathrm{P}, \mathrm{S}, \mathrm{Si}, \mathrm{Na}, \mathrm{Ca}, \mathrm{Ti}, \mathrm{Fe}, \mathrm{Al}, \mathrm{Cl}, \mathrm{Mg}, \mathrm{Mn}$, and $\mathrm{V}$. Excellent reviews of the physics of neutron - gamma ray techniques and how they are used for both remote and in situ geochemical analysis can be found in references [3-5].

\subsection{PING Timing Advantages}

The fact that different nuclear processes occur at different relative times between fast neutron pulses can be used to significantly increase both the accuracy and precision of the inferred elemental composition of planetary materials. The use of a properly designed PNG provides great flexibility in setting coincidence gates to optimize both data collection efficiency and spectral resolution, resulting in data with increased precision per gamma ray detected. The diagram in Figure 2 and spectra in Figure 3 show how restricting gamma-ray data acquisition to specially timed coincidence windows between neutron pulses results in the separation of gamma-ray spectra by nuclear process.

Bodnarik et al., (2012) [6], explain in detail how the coincidence time window boundaries and the PNG pulse width and period can be optimized to separate gamma ray lines from these different processes. Our calculation in section 4 makes use of this ability to correct for spectral contamination from different processes as discussed when computing the rate of the ${ }^{28} \mathrm{Si}$ inelastic line at $1779 \mathrm{keV}$. In the case of the $1779 \mathrm{keV}$ line there also happens to be delayed activation lines from both silicon and aluminum that result in a gamma ray at this same energy. Because the decay of these activated states are much longer than the PNG pulse, the $1779 \mathrm{keV}$ gamma rays from activation will persist into the time of the following fast neutron pulse and will thus contaminate the inelastic spectrum at this energy. We can correct for this contamination because the activation line intensities are essentially constant throughout the PNG pulse period. The activation line strength can be measured during the activation window and then scaled by the window widths so that its contribution can be subtracted from the count rate in the inelastic window. This concept is the basis for generating the "corrected" data described in section 4.

Figure 3 shows spectral data from a 6.3 hour run on the bare basalt in the experimental configuration described in section 3. Here we see the spectra from inelastic scattering, neutron capture and activation well separated and showing different gamma ray lines and different background levels.

\section{PING EXPERIMENTS}

\subsection{Description of the GGAO Test Site}

We are testing the capabilities of our PING instrument prototypes at a unique outdoor gamma ray and neutron instrumentation testing facility located at Goddard's Geophysical and Astronomical Observatory (GGAO) near the Goddard Space Flight Center main campus. A schematic aerial view of the test site is shown in Figure 4. This test facility allows us to operate PING on top of either of two large, assayed granite and basalt monuments, each $1.8 \mathrm{~m} \mathrm{x}$ $1.8 \mathrm{~m} \times 0.9 \mathrm{~m}$ in size. Activation Laboratories Ltd. in Ancaster, Ontario, Canada, has independently measured the full elemental compositions of these Concord Gray Granite and Columbia River Basalt materials to the ppm level. The

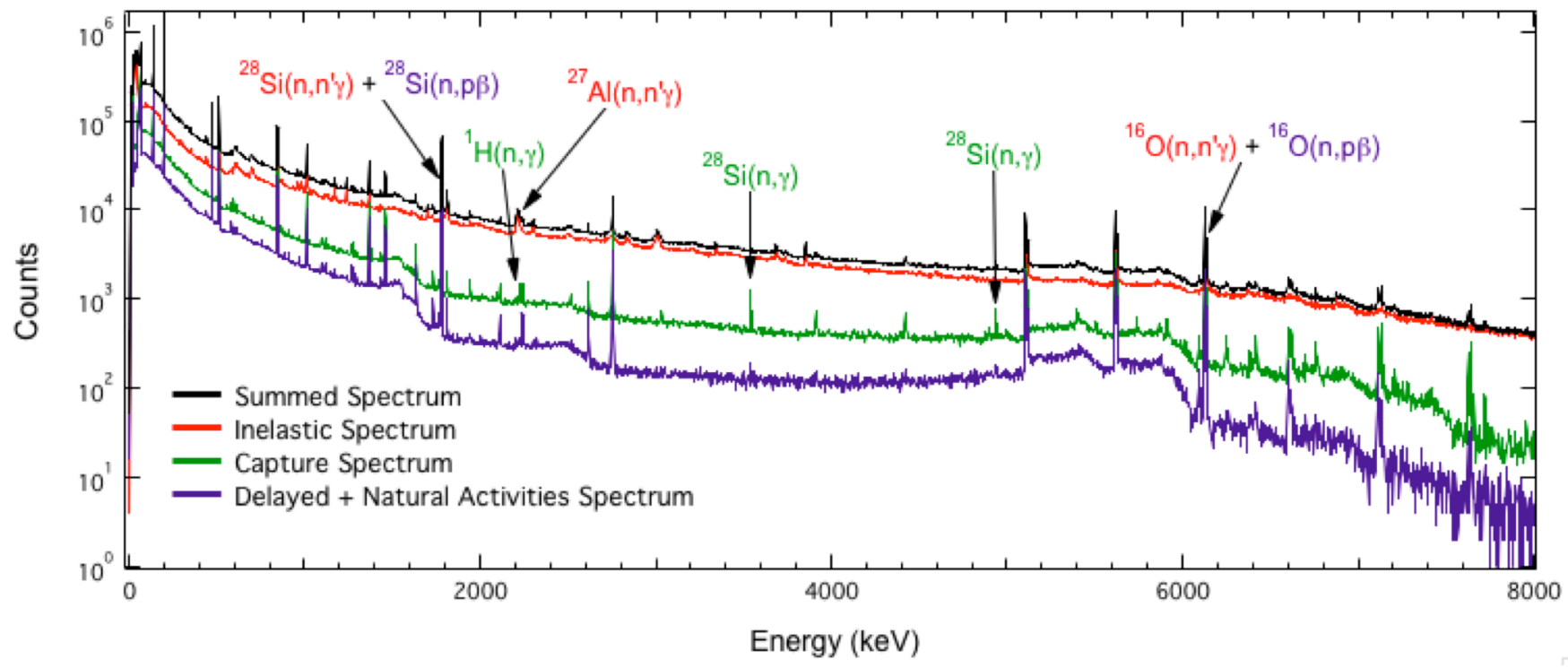

Figure 3. Spectra from different time windows: Gamma-ray spectra from a 6.33-hr acquisition using a HPGe detector on Columbia River basalt. 


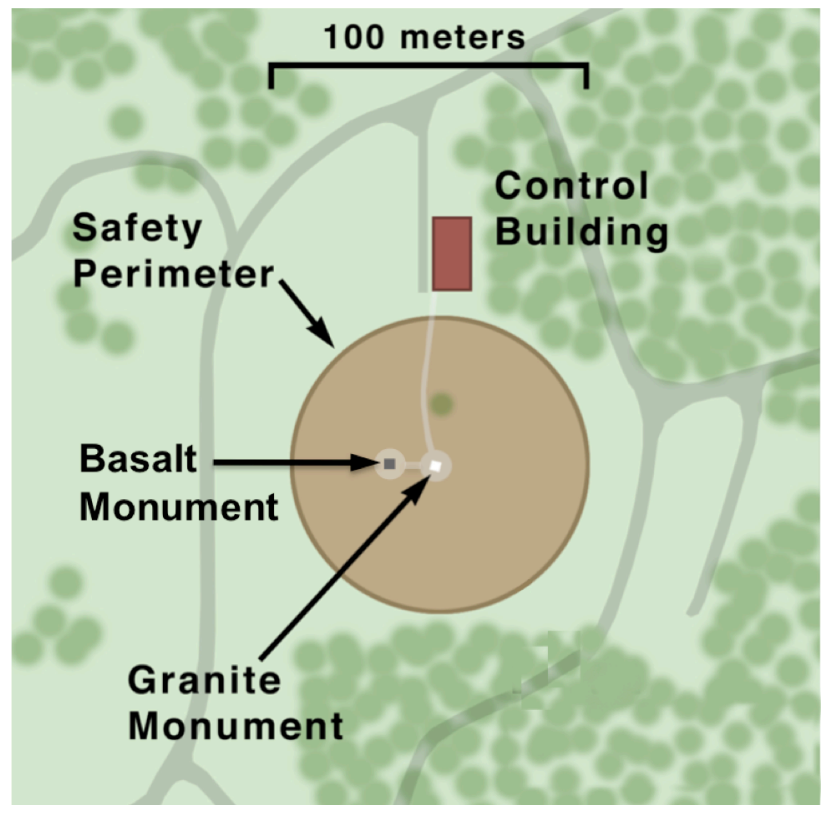

Figure 4. Aerial view of GGAO: This schematic of the outdoor gamma ray and neutron instrumentation testing facility shows the operations control building as well as the $46 \mathrm{~m}$ diameter safety perimeter surrounding the two existing $1.8 m \times 1.8 m \times 0.9 m$ granite and basalt monuments.

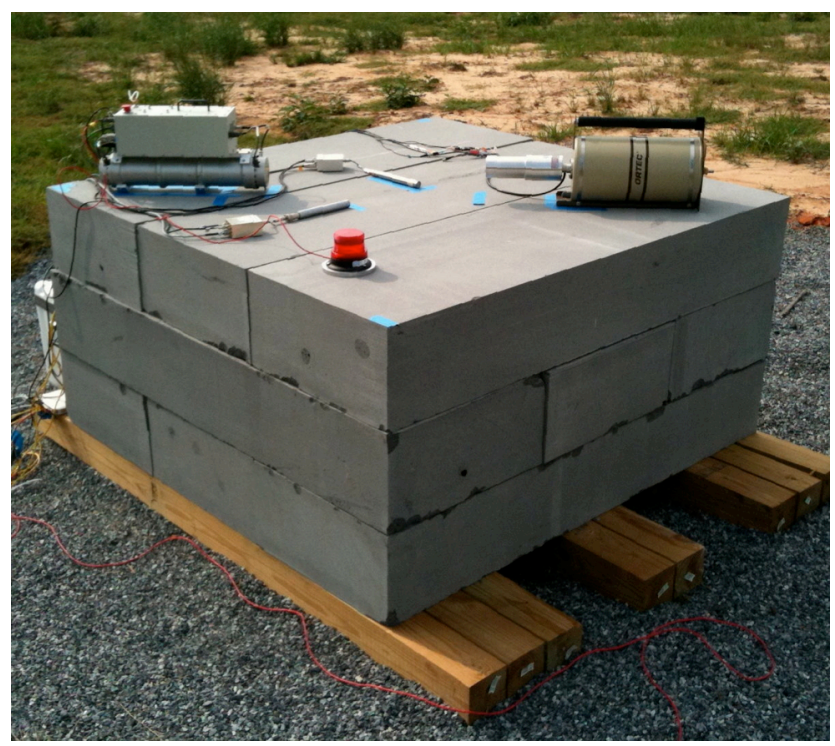

Figure 5. PING experiment set-up: PING deployed for measurements on top of the basalt monument. The PNG is on the left, the HPGe detector is on the right, and ${ }^{3} \mathrm{He}$ detectors are between them. The data acquisition electronics are situated behind the basalt and are not visible in this photo.

results of these assays are listed in Table 1. PING is remotely operated from a building $75 \mathrm{~m}$ from the monuments due to the radiation hazard from the PNG's 14 $\mathrm{MeV}$ neutrons. Underground power and communications lines connect the operations building to the test monuments.
Further information about the test facility can be found in $[7,8]$.

\subsection{The Importance of Using Both Granite and Basalt}

Both granite and basalt are igneous rocks containing large amounts of silicon and oxygen. While granite forms when magma cools slowly beneath a planet's crust, basalt forms when deeper magma breaks through the crust, such as through a volcano, and then cools quickly on the surface. Because basalts are formed from deeper magma, they contain significantly more of the heavier elements such as iron and magnesium than granite. When PING is able to measure the elemental composition of a planet's surface with sufficient precision to differentiate between igneous rock compositions, it will become a very effective tool for studying the composition of a planet's mantle as well as local igneous processes. The first step toward this goal is to test PING with both basalt and granite and make sure that it can differentiate between these extremes in iron composition. As our techniques mature and we learn to optimize PING for sensitivity to higher iron content, the precision in PING's material composition measurements will greatly improve.

\subsection{PING Experimental Setup}

Qualitative descriptions of PING results for various layering configurations such as with polyethylene and with the addition of materials such as titanium that are not found in either granite or basalt [1] have shown PING to be a powerful tool for subsurface elemental composition measurement. In this paper, we take the next step and begin the quantitative analysis of the elemental composition of the granite and basalt materials. We begin with a direct comparison between the bare basalt and bare granite configurations with PING resting directly on top of these large rock formations without any intervening layers of other materials. Figure 5 shows our experimental setup for the basalt. The identical configuration was used for the granite. Our PNG is a model MP320 from Thermo Scientific in Colorado Springs CO. Our gamma ray detector is an EG\&G ORTEC Gamma-Gage coaxial n-type high purity germanium (HPGe) gamma ray spectrometer connected to a portable liquid nitrogen dewar as shown in Figure 5. This detector is used to measure gamma rays in the $.05-10 \mathrm{MeV}$ range with an energy resolution of $0.2 \%$ at the $1779 \mathrm{keV} \mathrm{Si}$ inelastic gamma ray line. In this experimental configuration, both PNG and HPGe detector are placed directly on top of the granite and basalt monuments so that they rest on the opposite corners, aligned along the diagonal as shown in Figure 5. The PNG fast neutron emission point is located $100.5 \mathrm{~cm}$ from the flat face of the HPGe detector and is $9.5 \mathrm{~cm}$ above the surface of the monument. The cylindrical axis of the HPGe spectrometer is located $14.0 \mathrm{~cm}$ above the surface. The relative locations of the PNG and HPGe were identical for the tests on the basalt and granite. Neutron detectors were placed between the PNG and HPGe gamma ray spectrometer as shown in Figure 5. 
The PNG was set to emit an average of $0.4 \times 10^{8}$ neutrons per second with a pulse period of $1 \mathrm{~ms}$ and a100 $\mu$ s pulse duration. The gamma ray and neutron detectors were each read out in event-by-event mode by Lynx Digital Signal Analyzer units made by Canberra, Inc. using custom software. These data acquisition electronics were located below the surface of the formation so that the rock would provide shielding from the $14 \mathrm{MeV}$ fast neutrons. The Lynx and PNG electronic clocks were synchronized to the PNG neutron pulses via a TTL output pulse generated by the PNG. This synchronization ensures $0.1 \mu \mathrm{s}$ event time accuracy.

The preliminary data presented here represent 4 hours live time on both the basalt and the granite. The measured dead times were both $\sim 10 \%$.

\section{Preliminary Results}

\subsection{Comparing Data and Abundance Ratios}

As explained in section 2, the count rate for a gamma ray line that is due to a specific process on a single element is directly proportional to the abundance of that element in the material. Similarly, the equation below shows the linear relationship between the gamma ray line count rate ratio and the elemental abundance ratio.

$$
\frac{\omega_{A_{-} \text {Basalt }}}{\omega_{A_{-} \text {Granite }}}=\left(\frac{R_{A_{-} \text {Basalt }}}{R_{A_{-} \text {Granite }}}\right)\left(\frac{\sigma_{A_{-} \text {Basalt }} I_{\gamma A} \varepsilon\left(E_{A}\right)}{\sigma_{A_{-} \text {Granite }} I_{\gamma A} \varepsilon\left(E_{A}\right)}\right)
$$

Where

$\omega_{A_{-} \text {Basalt }} \omega_{A_{-} \text {Granite }}$ are the abundances of the same element $A$ in the basalt and in the granite (in wt \%)

$R_{A_{-} \text {Basalt }} R_{A_{-} \text {Granite }}$ are the count rates for a specific gamma ray line from element $A$ in the basalt and granite

$\sigma_{A_{-} \text {Basalt }}$ is the interaction cross section for element $A$

$I_{\gamma_{A}}$ is the number of gamma rays with energy $E_{A}$ emitted per neutron interaction

$\varepsilon\left(E_{A}\right)$ is the detector efficiency for gamma rays with energy $E_{A}$

Equation 1 is the result of the determination of the gamma ray count rate $\mathrm{R}$ for a given nuclear process involving a specific element. Equation 3 in Appendix A gives the count rate as a function of: 1) the macroscopic cross-section for both the bulk material and the specific element; 2) the number density of the specific element in the material; 3) the branching ratios for gamma ray emission of the specific line from a specific element; 4) detector efficiency, 5) PNG output, and 6) the absorption of emitted gamma rays as they travel through the rock to get to the gamma ray spectrometer. By taking the ratio of gamma ray rates for the same energy line from different formations, most of these factors cancel, especially considering that the basalt and granite densities are so similar $(2.69 \mathrm{~g} / \mathrm{cc}$ and $2.63 \mathrm{~g} / \mathrm{cc}$ respectively). In fact, most of the remaining factors in Equation 1 also cancel if we take the ratio of the intensity of the identical energy line in the basalt and in the granite experiments. Since our ratio concerns the same gamma ray line coming from the same element A but in the basalt vs. the granite, the remaining factors cancel and we are left with the simple relation:

$$
\frac{\omega_{A_{-} \text {Basalt }}}{\omega_{A_{-} \text {Granite }}}=\left(\frac{R_{A_{-} \text {Basalt }}}{R_{A_{-} \text {Granite }}}\right)
$$

The ratio of count rates from a specific gamma ray line from element A from basalt and granite is equal to the ratio of the abundance of element $A$ in the basalt and granite. (See Appendix A for a complete derivation of Equation 2.) In the following sections, we compute these ratios for the 1779 $\mathrm{keV}$ line from inelastic scattering on ${ }^{28} \mathrm{Si}$ and for the 2211 $\mathrm{keV}$ line from inelastic scattering on aluminum ${ }^{27} \mathrm{Al}$ and compare the ratio of rates to the ratios of the abundances of silicon to aluminum in the basalt and granite as given in Table 1. Table 2 contains the measured results needed for this comparison.

\begin{tabular}{|c|c|c|}
\hline \multirow{2}{*}{ Element } & $\begin{array}{c}\text { Columbia River } \\
\text { Basalt }\end{array}$ & $\begin{array}{c}\text { Concord Gray } \\
\text { Granite }\end{array}$ \\
\hline & Abundance (wt\%) & Abundance (wt\%) \\
\hline $\mathbf{O}$ & 44.97 & 48.59 \\
\hline Si & 23.18 & 34.59 \\
\hline Al & 8.637 & 7.32 \\
\hline $\mathbf{K}$ & 0.955 & 4.28 \\
\hline $\mathbf{N a}$ & 2.448 & 2.23 \\
\hline $\mathbf{F e}$ & 7.344 & 1.00 \\
\hline $\mathbf{C a}$ & 6.618 & 0.62 \\
\hline Mg & 4.794 & 0.17 \\
\hline $\mathbf{T i}$ & 0.898 & 0.14 \\
\hline $\mathbf{P}$ & 0.183 & 0.09 \\
\hline $\mathbf{H}$ & 0.087 & 0.00 \\
\hline Mn & 0.04 & 0.125 \\
\hline $\mathbf{R b}$ & 0.035 & 0.001 \\
\hline $\mathbf{C}$ & 0.03 & 0.03 \\
\hline $\mathbf{B a}$ & 0.026 & 0.036 \\
\hline Cl & 0.02 & 0.04 \\
\hline $\mathbf{Z r}$ & 0.014 & 0.016 \\
\hline $\mathrm{Ce}$ & 0.01 & 0.006 \\
\hline $\mathbf{N}$ & 0.01 & 0.01 \\
\hline Zn & 0.007 & 0.01 \\
\hline $\mathbf{S r}$ & 0.006 & 0.086 \\
\hline
\end{tabular}

Table 1: Granite and Basalt Assay Results 
Table 2: Measured Gamma Ray Count Rates with PING

\begin{tabular}{|c|c|c|c|c|c|c|c|}
\hline & Formation & $\begin{array}{c}\text { Egamma } \\
(\mathrm{keV})\end{array}$ & $\begin{array}{l}\text { Net Count } \\
\text { Rate }(\mathrm{cts} / \mathrm{s}) \\
\end{array}$ & $\begin{array}{l}\text { Net Count } \\
\text { Rate Error } \\
\text { (cts/s) }\end{array}$ & $\begin{array}{c}\text { Basalt/Granite } \\
\text { Ratio }\end{array}$ & $\begin{array}{c}\text { Basalt/Granite } \\
\text { Ratio Error } \\
\end{array}$ & $\begin{array}{l}\text { Independent } \\
\text { Assay Ratio }\end{array}$ \\
\hline${ }^{28} \mathrm{Si}\left(\mathrm{n}, \mathrm{n}^{\prime} \gamma\right)^{28} \mathrm{Si}$ & Basalt & 1779 & 0.664 & 0.037 & \multirow{2}{*}{0.707} & \multirow{2}{*}{0.101} & \multirow{2}{*}{0.670} \\
\hline${ }^{28} \mathrm{Si}\left(\mathrm{n}, \mathrm{n}^{\prime} \gamma\right)^{28} \mathrm{Si}$ & Granite & 1779 & 0.940 & 0.123 & & & \\
\hline${ }^{27} \mathrm{Al}\left(\mathrm{n}, \mathrm{n}^{\prime} \gamma\right)^{27} \mathrm{Al}$ & Basalt & 2211 & 1.089 & 0.019 & \multirow{2}{*}{0.936} & \multirow{2}{*}{0.023} & \multirow{2}{*}{1.180} \\
\hline${ }^{27} \mathrm{Al}\left(\mathrm{n}, \mathrm{n}^{\prime} \gamma\right)^{27} \mathrm{Al}$ & Granite & 2211 & 1.164 & 0.021 & & & \\
\hline
\end{tabular}

\subsection{Ratio of Si in Basalt to Si in Granite}

It would be most convenient to simply divide the gamma ray line count rates to get the abundance ratios. However, when there are times where counts are produced by more than a single reaction on a single element, there is no longer a linear relationship between the counts in the peak and the concentration of a single element. To get the most accurate result for an element's concentration it is necessary to remove the counts measured at times when they can be produced by multiple reactions or by different elements. One would like to have the $1779 \mathrm{keV}$ peak that occurs during the high-energy neutron pulse be only due to silicon. However, there is also peak at the same energy that is due to the delayed activity of aluminum. Since delayed activity peaks are present at all times, if these counts were not subtracted from the peak measured during the high-energy neutron pulse, the derived elemental concentration would be much too high.

Without the pulsing feature of our PNG and our use of timeresolved spectra, we would not be able to separate these processes. Because we can take gamma ray data during specific time windows between fast neutron pulses, we can isolate the contribution from the aluminum activation process and, after scaling by the ratio of time window widths, can subtract the contribution of this Al activation line from the $\mathrm{Si}$ inelastic line count rate. Using a $90 \mu \mathrm{s}$ inelastic window width and a $500 \mu \mathrm{s}$ absorption/activation window width for the basalt data and $85 \mu$ s and $475 \mu \mathrm{s}$ widths respectively for the granite data, we arrive at the following rates: for the ${ }^{28} \mathrm{Si} 1779 \mathrm{keV}$ line, the gamma ray rate is $0.664 \pm 0.037 \mathrm{cts} / \mathrm{s}$ from basalt, and $0.940 \pm 0.123$ $\mathrm{cts} / \mathrm{s}$ from the granite so that the count rate and hence the measured silicon concentration ratio is $0.707 \pm 0.101$. This measured value is to be compared with 0.670 as determined by the elemental assay data shown in Table 1 . We thus see good agreement between the measured and assay Si basalt to granite ratios; they agree to within one sigma.

\subsection{Ratio of Al in Basalt to Al in Granite}

To measure the aluminum abundance ratio between the basalt and granite, we will use the ${ }^{27} \mathrm{Al}\left(\mathrm{n}, \mathrm{n}^{\prime} \gamma\right)^{27} \mathrm{Al}$ inelastic scattering line at $2211 \mathrm{keV}$. By fitting the aluminum lines in the basalt and granite spectra, we measure $1.089 \pm 0.019$ $\mathrm{cts} / \mathrm{s}$ for basalt and $1.164 \pm 0.021 \mathrm{cts} / \mathrm{s}$ from granite, yielding a gamma ray count rate ratio, and thus aluminum concentration ratio of $0.936 \pm 0.023$. We note that this measured value is significantly lower than the 1.180 aluminum ratio calculated from the assayed abundances values shown in Table 1. It is not surprising that this aluminum ratio agreement isn't as good as the silicon because there is a fair amount of aluminum in the PNG and HPGe and neutron detectors that cannot be accounted for except by computer simulation. Thus, the aluminum measured by PING contains a constant value due to the PING equipment itself that must be added to the aluminum in the basalt or granite information and thus cannot be divided out in the ratio. Adding the same constant factor to both the numerator and denominator will reduce the ratio so that the result will be less than the expected ratio of the elemental assay data. This problem will be eliminated when our full Monte Carlo is running as described in Section 5 below.

\section{FUTURE WORK}

In the future, these data will be coupled with MCNPX [9] computer simulations to allow us to quantitatively determine the bulk elemental composition of the subsurface material for any solid body in the solar system. The MCNPX calculations allow an exact statistical calculation of both the energy and the time of a gamma-ray event detected in our gamma ray spectrometer. The calculations take into account all the details of neutron production and transport, all nuclear reactions on all elements present in the material, and all possible gamma rays that can be produced and their transport and detection at a specific point in space by a particular detector. Thus, the Monte Carlo calculations will provide a direct relationship between peak counts and elemental concentrations, limited only by the statistical count rate uncertainty and the systematic calculation uncertainties (generally less than 1\%).

Many of the parameters in the equations in section 4 and in Appendix A can only be determined using MCNPX computer simulations. The process by which gamma rays are generated and detected by the spectrometer is not analytically tractable. Indeed, not only are the neutrons emitted isotropically and can move around inside the formation material as they are moderated but they also emit gamma rays isotropically. A small fraction of the gamma rays will be going in the right direction to be intercepted by 
the detector if they aren't absorbed by the rock before they get there. Finally, many of the gamma rays that enter the detector will pass through it without being detected. The computer simulation traces all of these processes and includes the physics of neutron transport and interaction.

Since landed planetary science instruments are limited by mass, power and volume, it is unlikely that it would be possible to use an HPGe gamma ray spectrometer for an actual mission due to the bulk and power required by its cryogenics system. Actual space-flight versions of PING would use spectrometers such as scintillators that have poorer energy resolution. We used the high resolution HPGe in these tests with known material samples so that we could best characterize the underlying spectra for the granite and basalt materials and thus better understand and characterize spectra taken with detectors with poorer energy resolution. The precision of elemental measurements is improved for increased exposure time. The chosen integration time for a measurement of a particular element is governed by the quantity of that element in the planetary soil and the measurement precision required.

\section{Conclusions}

We have measured the basalt to granite ratio for silicon with good agreement to the independent assay results. We have also measured the basalt to granite aluminum ratio with some success, especially considering that some of the measured aluminum comes from PING itself. The method described for comparing the elemental composition between the basalt and granite is not limited to the silicon and aluminum examples shown here. Using this procedure we can infer the basalt to granite ratio for the other major elements to arrive at a comparison of the total composition to within a few percent. The addition of Monte Carlo simulations will allow us to make independent quantitative measurements of elemental composition by performing the calculation of analytically intractable neutron and gamma ray transport factors that cancel in the ratio.

This paper has shown PING to be a promising technique for in situ elemental abundance analysis of materials. Continued work to refine the technique and to build more flight-like prototypes will make it accessible to future flight opportunities for in situ measurements on any rocky body in the solar system.

\section{REFERENCES}

[1] A. Parsons, et al., Nucl. Instr. and Meth. A 652, 2011, 674.

[2] C. Pieters and P. Englert (Eds.), Remote Geochemical Analysis: Elemental and Mineralogical Composition, Cambridge University Press, 1993.

[3] L.G. Evans, R.C. Reedy and J. I. Trombka in C. Pieters and P. Englert (Eds.), Remote Geochemical Analysis: Elemental and Mineralogical Composition, Cambridge University Press, 1993, 167-198.

[4] J. Grau, J. Schweitzer, and R.C. Hertzog, IEEE Trans. Nucl Sci., 31, 1990, 1-4.

[5] W.V. Boynton, L.G. Evans, R.C. Reedy and J.I. Trombka in C. Pieters and P. Englert (Eds.), Remote Geochemical Analysis: Elemental and Mineralogical Composition, Cambridge University Press, 1993, pp. 395-411.

[6] J. G. Bodnarik et al., Nucl. Instr. and Meth. A., 2013, http://dx.doi.org/10.1016/j.nima.2012.12.110

[7] J. Bodnarik, et al., 41 ${ }^{\text {st }}$ LPSC, Woodlands, TX, 1-5 Mar., 2010; Conf. Proc. 41 (2010) 2581.

[8] A. Parsons, et al., 2011 IEEE (NSS/MIC), Valencia, Spain, 23-29 Oct., 2011;

[9] Pelowitz, D. ed., (2005), MCNPX User's Manual, LANL 
Biographies

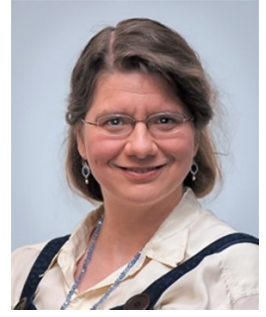

Ann Parsons received a B.S. in Physics from University of Michigan, Ann Arbor in 1986 and a Ph.D in Physics from U.C. Berkeley in 1993. Dr. Parsons has been with NASA/ Goddard Space Flight Center as a staff scientist for over 17 years. During her tenure at Goddard, Dr. Parsons has been the Detector Scientist for the Burst Alert Telescope on the Swift Gamma Ray Burst Explorer launched in 2004 and has since been working on novel scientific instrumentation for planetary science applications. She is currently the head of the Planetary Geochemistry Flight Instrumentation Group in Goddard's Solar System Exploration Division.

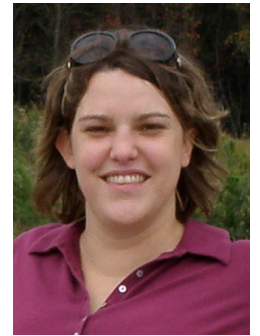

Julia Bodnarik received her $B S$ in Physics from Wichita State University in 2002, her MA in Physics from Fisk University in 2007 and Ph.D in physics from Vanderbilt University in early 2013. Ms. Bodnarik's experience includes work at both the Gemini North Observatory and the Smithsonian Astrophysical Observatory Submillimeter Array on Mauna Kea, Hawaii (2002-2005). Her doctoral research has been performed at NASA/ Goddard Space Flight Center on the development of coupled pulsed neutron generator, gamma ray and neutron detectors instrumentation for in situ planetary surface elemental composition analysis.

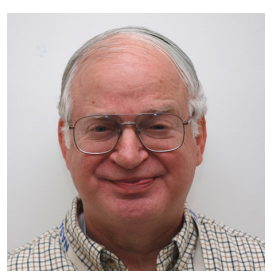

Larry Evans is Senior Principal Scientist for Science Programs at Computer Sciences Corporation and been funded to participate in research at NASA/ Goddard Space Flight Center since 1973. He has been either a participating scientist or a science team member for the Mars Observer Mission Gamma-Ray Spectrometer, the Near Earth Asteroid Rendezvous (NEAR) X-ray/Gamma-ray Spectrometer, Mars Odyssey Orbiter, as well as Deputy GRS Instrument Scientist for the MESSENGER mission to Mercury. Currently Co-Investigator for the LEND Instrument on the Lunar Reconnaissance Orbiter (LRO) Dr. Larry Evans received his Ph.D. in Applied Physics from Northwestern University in 1971.

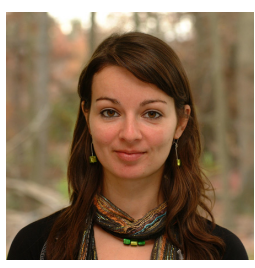

Suzanne Nowicki received her BS in Physics from Université Joseph Fourier, Grenoble, France in 2004. She earned MSE degrees in both Engineering Electronics, from ENSICAEN, in Caen, France (2007) and in Nuclear Engineering and Radiological Sciences, from the University of Michigan, Ann Arbor, MI (2009). Ms. Nowicki is soon to finish her Ph.D in Applied Physics at the University of Michigan based on research performed at NASA/ Goddard Space Flight Center on the development and adaptation of pixelated CdZnTe gamma-ray imaging technology for in situ planetary science applications.

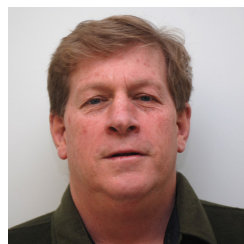

Timothy McClanahan has been a Computer Scientist with NASA/ Goddard Space Flight Center for over 15 years and specializes in signal processing and image analysis of data from X-ray, gamma ray and neutron planetary science missions. His involvement includes analysis for the Mars Odyssey Gamma-ray Spectrometer, the Near Earth Asteroid Rendezvous, (NEAR), X-ray and Gamma-ray Spectrometers (XGRS) and LRO-LEND neutron detector development and signal analysis. He is currently Lead Ground Systems Engineer for the Voyager 1, 2 missions. Dr. McClanahan received a BS from Northern Arizona University and both masters and doctoral degrees in computer science from George Mason University.

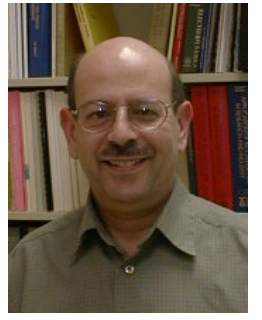

Jeff Schweitzer has been a Research Professor of Physics at the University of Connecticut, since 1997 where he performs research in nuclear physics and astrophysics, radioactive beams; planetary measurements; solar physics; detector research; applied nuclear physics, esp. in geological, forensic, nanoscale NRRA for material analysis and cement hydration reactions, medical and industrial applications. He worked at Schlumberger-Doll Research from 1974-96 studying nuclear geophysics; nuclear astrophysics; detector research; planetary measurements. He has worked at the California Institute of Technology and received his Ph.D. in physics from Purdue University in 1972 studying nuclear physics and first-forbidden beta decay. 


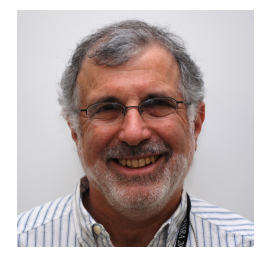

Richard Starr is a Research Associate Professor of Physics at The Catholic University of America and has been funded through NASA grants to conduct $X$-ray, gamma-ray, and neutron studies at the Goddard Space Flight Center in Greenbelt, MD since 1980. He has worked on such flight programs as the GRS for Mars Observer, the X-Ray/Gamma-Ray Spectrometer (XGRS) for the Near Earth Asteroid Rendezvous Shoemaker (NEAR) mission, the Russian Mars '96 GRS, the X-Ray Spectrometer for the Clark mission and the GRS instrument suite on the 2001 Mars Odyssey mission. He is currently the X-Ray Spectrometer instrument scientist and participating scientist for the Mercury MESSENGER mission, and as a co-investigator on the Lunar Exploration Neutron Detector (LEND) on the Lunar Reconnaissance Orbiter. 


\section{APPENDIX A}

\section{Derivation of the Ratio of Elemental Abundances as a Function of Count Rate Ratio}

We start with the equation describing the detector count rate from a specific gamma ray emitted by element A in any material. The following equation is appropriate for inelastic scattering interactions such as those that produced the silicon $1779 \mathrm{keV}$ and aluminum $2211 \mathrm{keV}$ lines used in the calculations shown in this paper:

$R_{A}=n_{A} \sigma_{A} N I_{\gamma A} \varepsilon\left(E_{A}\right) P_{\gamma A}$

where

$R_{A}$ is the count rate for a specific gamma ray line from element $\mathrm{A}$

$n_{A}$ is the number density of element $\mathrm{A}$ in the material

$\sigma_{A}$ is the inelastic scattering cross section for element $\mathrm{A}$

$N$ is the rate of neutron interactions and is proportional to the neutron generator output rate

$I_{\gamma_{A}}$ is the number of gamma rays with energy $E_{A}$ emitted per neutron interaction

$\varepsilon\left(E_{A}\right)$ is the detector efficiency at energy $E_{A}$

$P_{\gamma_{A}}$ is the average gamma ray absorption probability for all gamma ray paths through the material

Some of the above factors require Monte Carlo calculations due to complex geometry. However, they cancel out when you take the ratio:

$$
\frac{R_{A_{-} \text {Basalt }}}{R_{A_{-} \text {Granite }}}=\frac{n_{A_{-} \text {Basalt }} \sigma_{A} N I_{\gamma A} \varepsilon\left(E_{A}\right) P_{\gamma A_{-} \text {Basalt }}}{n_{A_{-} \text {Granite }} \sigma_{A} N I_{\gamma A} \varepsilon\left(E_{A}\right) P_{\gamma A_{-} \text {Granite }}}
$$

Where $n_{A_{-} \text {material }}=\frac{\omega_{A_{-} \text {material }} \rho_{\text {material }} N_{\text {Avogadro }}}{A_{A}}$

Here $A_{A}$ is the atomic mass of element A, $N_{\text {Avogadro }}$ is Avogadro's number, $\rho$ is the material density and

$\omega_{A}$ is the abundance of element $A$ in each material.

Substituting for $n_{A}$ we have:

$$
\frac{R_{A_{-} \text {Basalt }}}{R_{A_{-} \text {Granite }}}=\left(\frac{\omega_{A_{-} \text {Basalt }}}{\omega_{A_{-} \text {Granite }}}\right) \frac{A_{A} \rho_{\text {Basalt }} N_{\text {Avogadro }} \sigma_{A_{-} \text {Basalt }} N I_{\gamma A} \varepsilon\left(E_{A}\right) P_{\gamma A_{-} \text {Basalt }}}{A_{A} \rho_{\text {Granite }} N_{\text {Avogadro }} \sigma_{A_{-} \text {Granite }} N I_{\gamma A} \varepsilon\left(E_{A}\right) P_{\gamma A_{-} \text {Granite }}}
$$

Since the densities of basalt and granite are very similar and the probability of absorption $P$ of the same gamma ray with energy $E_{A}$ will be the same in both materials, both of these factors cancel in the ratio. The input neutron factor $N$ as well as Avogadro's number will also cancel. Solving for the abundance ratio we arrive at Equation 1 in the text:

$$
\frac{\omega_{A_{-} \text {Basalt }}}{\omega_{A_{-} \text {Granite }}}=\left(\frac{R_{A_{-} \text {Basalt }}}{R_{A_{-} \text {Granite }}}\right)\left(\frac{\sigma_{A_{-} \text {Basalt }} I_{\gamma A} \varepsilon\left(E_{A}\right)}{\sigma_{A_{-} \text {Granite }} I_{\gamma A} \varepsilon\left(E_{A}\right)}\right)
$$

The detector efficiencies and branching ratio $I$ are independent of the formation material and also cancel in the ratio. Finally the cross sections for gamma ray energy $E_{A}$ will cancel because we are considering the case where the gamma ray comes from the same element. 
We are finally left with Equation 2:

$$
\frac{\omega_{A_{-} \text {Basalt }}}{\omega_{A_{-} \text {Granite }}}=\left(\frac{R_{A_{-} \text {Basalt }}}{R_{A_{-} \text {Granite }}}\right)
$$

Note that for gamma ray rates from lower energy neutron capture interactions, the formula is slightly different. The cross section for interaction must be represented as the fractional macroscopic neutron capture cross section in the material. We thus start with the following equation:

$R_{A_{-} \text {Basalt }}=\left(\frac{n_{A_{\_} \text {Basalt }} \sigma_{A}}{\sum_{i=1}^{\# \text { elements }} n_{i_{-} \text {Basalt }} \sigma_{i}}\right) N I_{\gamma A} \varepsilon\left(E_{A}\right) P_{\gamma A_{-} \text {Basalt }}$

Where all other factors are as defined on the previous page except that

$\sigma_{A}$ is now the neutron capture cross section for element $A$ and

\# elements

$\sum_{i=1}^{\# \text { elements }} n_{i_{-} \text {Basalt }} \sigma_{i}$ is the total macroscopic cross section for neutron interactions

We make the excellent approximation that $\sum_{i=1}^{\text {\#elements }} n_{i_{-} \text {Basalt }} \sigma_{i} \approx \sum_{i=1}^{\# \text { elements }} n_{i_{-} \text {Granite }} \sigma_{i}$ so that this factor cancels in the ratio. The calculation then proceeds identically to that for the inelastic scattering interactions shown on the previous page. 